\title{
Posterior Cervical Pedicle Screw-Rod/Plate Instrumentation Combined with Unilateral Open-Door Laminoplasty for the Treatment of Acute Cervical Spinal Cord Compression Injury: Report of Five Cases
}

\author{
Li ZHANG ${ }^{1}$, Yu SUN ${ }^{1}$, Yongchong JIANG ${ }^{2}$, Yong WANG ${ }^{3}$ \\ ${ }^{1}$ Peking University Third Hospital, Department of Orthopedic Surgery, Beijing, China \\ ${ }^{2}$ The First Hospital of Qinhuangdao, Department of Orthopedic Surgery, Qinhuangdao, China \\ ${ }^{3}$ Beijing North Hospital of Ordnance Industry, Department of Surgery, Beijing, China
}

\section{ABSTRACT}

All patients were acute, multi-segment cervical spinal cord compression injury secondary to subaxial cervical spine fracture. These patients underwent emergency posterior cervical pedicle screw-rod/plate instrumentation combined with unilateral open-door laminoplasty. Well-placed pedicle screws and good cervical canal dilation were achieved in all patients, as assessed by radiography. Patients were followed up for 6-10 months and showed at least one class improvement in spinal impairment. These cases suggest that simple posterior cervical pedicle screw-rod/plate instrumentation combined with extensive unilateral open-door laminoplasty could repair acute, multi-segment cervical spinal cord compression injury secondary to subaxial cervical spine fracture if the cervical spine sequence is well preserved and does not require restoration.

KEYWORDS: Subaxial cervical spine fracture, Multi-segment spinal cord compression, Pedicle screw, Laminoplasty

\section{INTRODUCTION}

$\mathrm{I}$ $\mathrm{t}$ is technically challenging to manage a multi-segment cervical spinal cord compression injury secondary to subaxial cervical spine fracture (5). Commonly used techniques include anterior cervical spinal cord decompression (corpectomy) and screw-plate instrumentation, as well as posterior decompression with lateral mass fixation (5).

Anterior subtotal corpectomy and screw-plate instrumentation with bone grafting or titanium mesh interbody fusion (IBF) is effective for treating single-level cervical vertebra body fracture (5). However, anterior multi-segment IBF has been associated with a high risk of failure in patients with multisegment fractures, with long bone graft or titanium mesh implantation (12). Anterior instrumentation might carry a higher risk of surgical morbidities (3).
We describe the surgical technique, clinical outcome and follow-up of five patients with acute cervical compression $\mathrm{SCl}$ secondary to subaxial cervical spine fracture who underwent posterior cervical pedicle screw-rod or screwplate instrumentation combined with unilateral open-door laminoplasty.

\section{CASE REPORTS}

All five patients were male, median age 53 years (range 2660) (Table I) who were hospitalized for multi-segment cervical compression $\mathrm{SCl}$ secondary to cervical spine fracture. Three of 5 patients showed an ASIA class of B prior to surgery and 2 of 5 patients was classified as ASIA class $C$. 


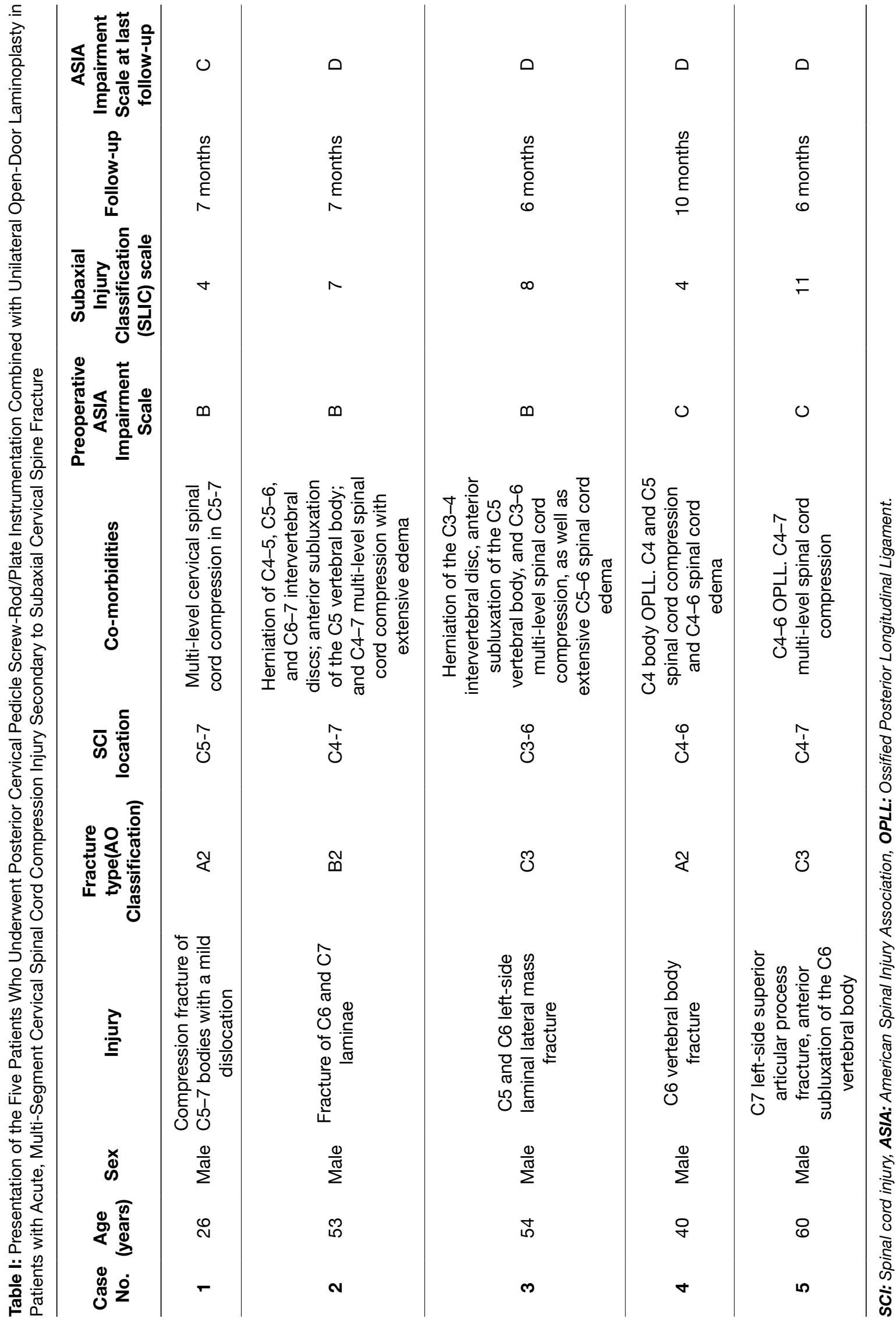




\section{Surgical Technique}

The patient was positioned prone using a Mayfield head clamp. The neck was placed in the neutral flexion-extension position. A posterior median incision was made to expose the spinous processes, laminae and lateral masses of the target segments, and the spinous processes were trimmed for further bone harvest. Cervical pedicle screws were manually inserted at a length of 26-28 mm using the anatomical positioning method under the guidance of lateral fluoroscopy (7). The cartilage surface of the articular facet joint to be fixed was removed with a rongeur and curette, and the fragmented bones were implanted. An appropriate-sized rod or plate was pre-curved to the physiological cervical curvature and was tightened in situ. The pedicle screws were all fixed on the normal vertebral body. For vertebral body fracture, an additional pedicle screw was inserted in the unilateral pedicle, while the side of vertebral body and pedicle were left intact. The bone fracture lines involving the bilateral sides of a vertebral body were left unfixed. Multi-segment, open-door unilateral laminoplasty was concomitantly performed according to the magnetic resonance imaging $(\mathrm{MRl})$ results demonstrating the degree and range of spinal cord compression. Normally, 4-6 laminae were decompressed with one extra-decompressed segment beyond the compressed segment, both superiorly and inferiorly. A \#10 silk suture (Shanghai Medical Suture Needle Factory Co., LTD, Shanghai, China) was pulled through a hole drilled in the root of the spinous process onto the hinged-side articular sac or tied underneath the hinged-side rod or plate.

\section{Outcome}

Four of 5 patients (Case 2: Figure 1A-D; Case 4: Figure 2A-D) showed an ASIA class of D and 1 patient was ASIA class $C$ (Table I). No patient exhibited worsening neurological symptoms, and none complained of dizziness, headache, consciousness alteration, hemiplegia or other vertebral-basilar artery ischemia-associated symptoms. Postoperative anteroposterior and lateral cervical radiography revealed well-placed screws and opened spinal canals in all patients. No compli- cation occurred. All five patients exhibited improvements in limb neurological motor-sensory function during follow-up as detailed below.

\section{DISCUSSION}

This case series describes the indications, operative technique and treatment outcome of posterior cervical pedicle screw$\mathrm{rod} /$ plate instrumentation combined with unilateral open-door laminoplasty in patients with acute, multi-segment cervical spinal cord compression injury secondary to subaxial cervical spine fracture.

Multi-segment spinal cord compression injury is normally refractory to conventional surgery. Lateral mass screw-plate or screw-rod instrumentation has generally been used for posterior cervical spine fixation and fusion. Insertion of lateral screw required a $10^{\circ}$ to $25^{\circ}$ camber and resulted in a relatively medial placement of the screw tail, which could interfere with laminoplasty. Therefore, in most cases laminoplasty has to be performed prior to the lateral screw insertion and screwrod instrumentation. However, since the spinal dura mater is already exposed during the laminoplasty, a catastrophic result of spinal cord injuries may be caused by surgical instruments or tools slipping into the spinal canal (15), which has restricted the use of posterior cervical laminoplasty combined with lateral mass fixation. In addition, failed screw insertion is incurable due to the small volume of lateral mass, and is more frequent in patients with osteoporosis.

Cervical pedicle screw instrumentation achieved greater biomechanical stability than any other cervical internal fixation techniques, including anterior plating combined with posterior lateral mass fixation (8). Pedicle screw placement requires an approximately $45^{\circ}$ angle with the screw tail laterally located in order to prevent any interference with subsequent laminoplasty. This allowed the screw-rod instrumentation to occur prior to the laminoplasty and consequently minimized the risk of iatrogenic spinal cord injury (SCl) caused by instrument or tools impingement (16). The fixation range of the posterior pedicle
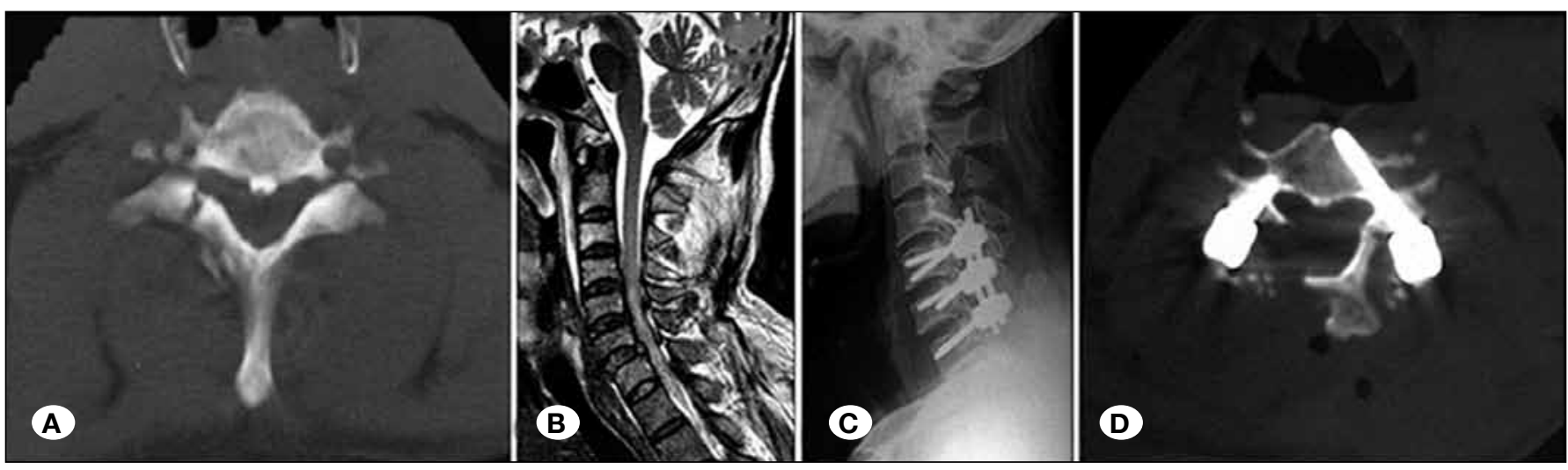

Figure 1: A 53-year-old male suffered from C6-7 lamina fracture as shown on CT (A), with complicating herniation of the C4-7 discs, C4-7 multi-level spinal cord compression and extensive spinal cord edema on MRI (B). ASIA was Class B. The patient underwent C5-7 pedicle screw instrumentation and posterior C3-7 unilateral open-door laminoplasty (C and D) 10 days after SCI. Follow-up at 7 months showed that the neurological function was improved to ASIA Class D. 

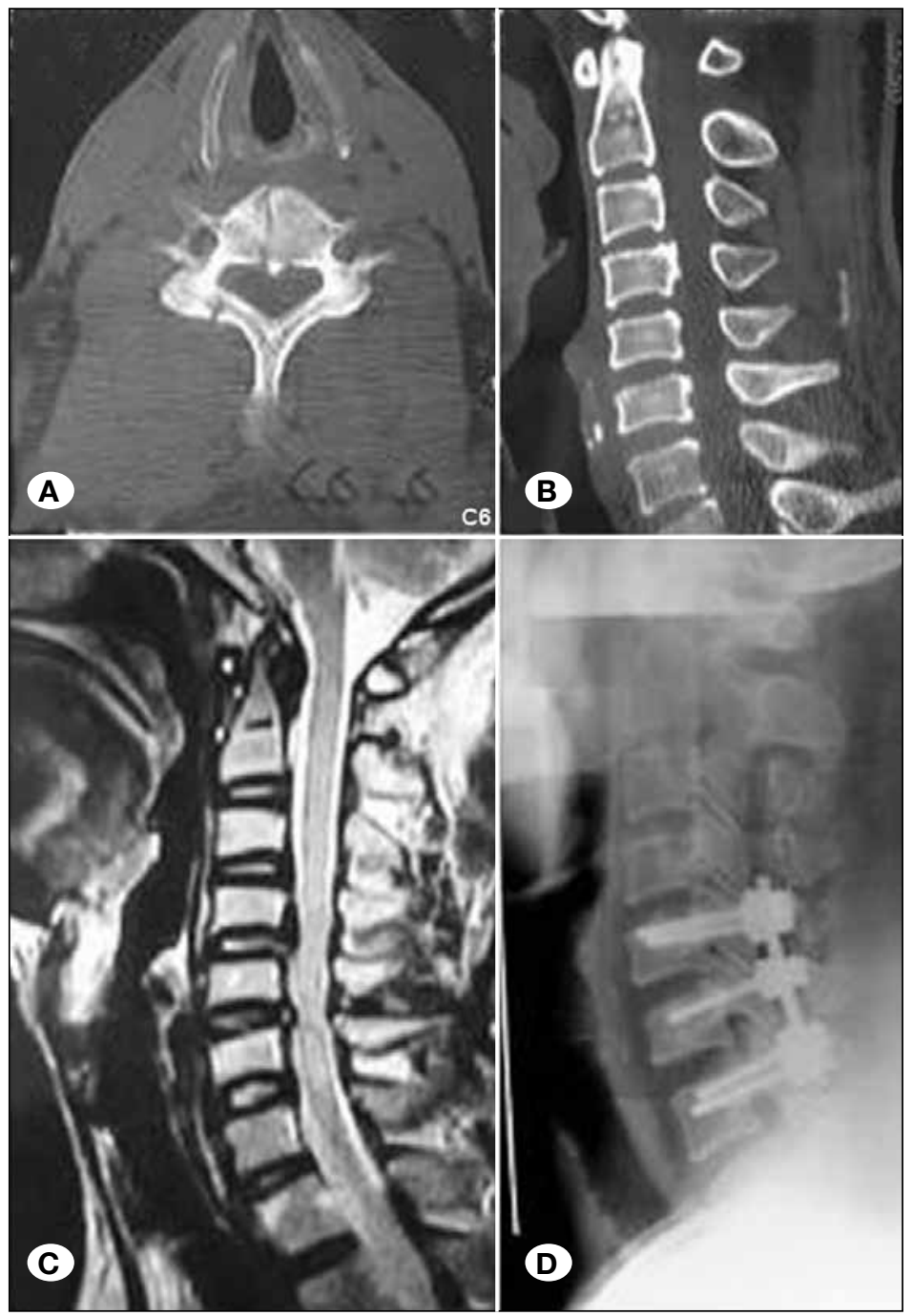

Figure 2: A 40-year-old male experienced acute fracture of the C6 vertebrae without displacement (A) and OPLL of C4 (B) with multiple segment compression, cervical spinal cord edema (C), and was ASIA Class D. Surgery: C3-7 unilateral open door laminoplasty and C4-T1 pedicle instrumentation (D). Because the left side of the C6 pedicle was intact, we inserted an additional pedicle screw into the fractured $\mathrm{C} 6$ to enhance instrumentation stiffness. Follow-up at 10 months after surgery did not show that the neurologic function was improved, and an ASIA class D was achieved.

screw was dependent on the extension of the vertebral body or laminal fracture involving non-fractured, stable vertebra in a relatively flexible way from $\mathrm{C} 2$ toward the thoracic vertebrae. Liu et al. reported the outcomes of 25 patients with cervical spine instability undergoing posterior C3 - C7 single/double door laminoplasty and free-hand cervical pedicle screw (CPS) fixation as well as graft fusion (9). During the follow-up, fused segments were stable and no neurovascular complications were observed.

Pedicle screw instrumentation combined posterior laminoplasty might provide sufficient decompression and cervical stability for cervical spondylotic myelopathy patients with multi-segment spinal cord compression and cervical instability $(6,16)$. In the posterior approach, a single incision allowed concomitant decompression and fixation without any procedural conflict. Using bone harvest from spinous processes allowed surgery to be performed at only one place, without the need to harvest iliac bone. For cervical spondylotic myelopathy accompanying local kyphosis or segmental instability, combined procedure of posterior reconstructive surgery using a pedicle screw instrumentation provided better clinical outcomes than laminoplasty alone (1). As in a previous study (10), our patients achieved a good recovery.

In case of reactive edema of spinal cord after SCl, there is an increase in the transverse diameter of the cervical spinal cord, which could result in further spinal cord compression (11). Posterior extensive laminoplasty can achieve greater decompression compared with its anterior counterpart (4). However, for patients with extensive spinal cord edema, anterior decompression can hardly achieve good outcomes since patients' spinal cords are extensively compressed (13). Concomitant laminectomy can eliminate the compression induced by fractured lamina on the cervical spinal cord in the presence of multi-segment lamina fracture.

Early stable cervical immobilization accelerates cervical $\mathrm{SCl}$ recovery and facilitates airway management (17). Cervical pedicle screw-rod instrumentation achieved a better biomechanical stabilizing effect than other cervical internal fixation techniques (8). It was reported that cervical spondylotic myelopathy secondary to multi-segment cervical spinal cord 
compression was better treated using posterior laminoplasty as opposed to anterior cervical decompression (14).

For cases of subaxial cervical spine fractures, pedicle screwrod instrumentation combined with laminoplasty is suitable for those with multi-segment fractures and spinal cord compressions or those with cervical fractures complicated by developmental or degenerative cervical stenosis and multi-segment spinal cord compressions, and in particular those with lateral mass fractures (they can not take lateral mass screw-rod instrumentation) and those suffering from osteoporosis (lateral mass screw-rod instrumentation often fails in such patients); however, for cases of single segment spinal cord compression or burst fractures, anterior cervical corpectomy and fusion is recommended.

There were limits of this approach.

Clinicians worry about the possible risks of damages to the spinal cord, nerve root and vertebral artery caused by vertebral pedicle screw instrumentation with free hands; however, Abumi et al. reported their experiences of 712 screws manual insertion into the cervical pedicle and the complication of that was no difference with cervical lateral mass screw insertion. (2), and a significantly lower incidence rate of screw loosening in the former, after comparing the complications related to vertebral pedicle screw instrumentation and those associated with cervical lateral mass plate fixation were reported by the correlative studies. However, for patients with vertebral artery abnormalities or scleroses, injuries of the vertebral artery and the secondary risk of brainstem infarction should also be concerned during pedicle screw instrumentation; therefore, vertebral artery abnormalities or scleroses are the contraindications of this approach, while CTA (CT angiogram) or MRA (MR angiogram) for the vertebral arteries should be performed preoperatively to exclude abnormalities or scleroses of the vertebral artery. In addition, for patients with vertebral pedicle fracture, pedicle screw instrumentation cannot be done either.

Vertebral pedicle screw instrumentation with free hands is a hard technique to the surgeons. There will be a learning curve for them to gain considerable experiences before they can judge the accuracy of screw instrumentation. Moreover, since the head end metal frame of the operating table will shelter the X-ray, anteroposterior radiographs are unavailable intraoperatively and experienced surgeons are required to judge the accuracy of screw insertion based on the lateral radiographs and other indices. In addition, since lateral $\mathrm{X}$-rays are shelter by the shoulder, screws fixated at the C7-T1 levels can hardly be revealed, which again makes the experienced surgeons a necessity during judging the accuracy of screw instrumentation based on the other indices.

\section{- CONCLUSION}

For patients with preserved cervical alignment, simple posterior cervical pedicle screw-rod or screw-plate instrumentation with extensive laminoplasty is an option treatment for subaxial cervical multi-segment vertebral body or lamina fracture or single-segment vertebral body fracture complicated by multisegment spinal cord compression and degenerative cervical stenosis. Further larger studies are necessary to assess the efficacy and safety of this approach.

\section{ACKNOWLEDGEMENT}

The authors thank the patients for their active cooperation, and the other orthopedic doctors and nurses for their hard work. Thanks to Zhenhui Zhang for help with data collection.

\section{REFERENCES}

1. Abumi K: Cervical spondylotic myelopathy: Posterior decompression and pedicle screw fixation. Eur Spine J 24 Suppl 2:186-196,2015

2. Abumi K, Shono $\mathrm{Y}$, Ito $\mathrm{M}$, Taneichi H, Kotani $\mathrm{Y}$, Kaneda K: Complications of pedicle screw fixation in reconstructive surgery of the cervical spine. Spine (Phila Pa 1976) 25:962969,2000

3. Acosta FL Jr, Aryan HE, Chou D, Ames CP: Long-term biomechanical stability and clinical improvement after extended multilevel corpectomy and circumferential reconstruction of the cervical spine using titanium mesh cages. J Spinal Disord Tech 21:165-174, 2008

4. Cabraja M, Abbushi A, Koeppen D, Kroppenstedt S, Woiciechowsky C: Comparison between anterior and posterior decompression with instrumentation for cervical spondylotic myelopathy: Sagittal alignment and clinical outcome. Neurosurg Focus 28: E15, 2010

5. Consortium for Spinal Cord Medicine: Early acute management in adults with spinal cord injury: A clinical practice guideline for health-care professionals. J Spinal Cord Med 31:403-479, 2008

6. Demura S, Murakami H, Kawahara N, Kato S, Yoshioka $\mathrm{K}$, Tsuchiya $\mathrm{H}$ : Laminoplasty and pedicle screw fixation for cervical myelopathy associated with athetoid cerebral palsy: Minimum 5-year follow-up. Spine (Phila Pa 1976) 38:17641769, 2013

7. Jeanneret B, Gebhard JS, Magerl F: Transpedicular screw fixation of articular mass fracture-separation: Results of an anatomical study and operative technique. J Spinal Disord 7: 222-229, 1994

8. Kothe R, Ruther W, Schneider E, Linke B: Biomechanical analysis of transpedicular screw fixation in the subaxial cervical spine. Spine (Phila Pa 1976) 29:1869-1875, 2004

9. Liu Y, Hu JH, Yu KY: Pedicle screw fixation for cervical spine instability: Clinical efficacy and safety analysis. Chin Med J (Engl) 122: 1985-1989, 2009

10. Ni B, Zhou F, Guo Q, Li S, Guo X, Xie N: Modified technique for C1-2 screw-rod fixation and fusion using autogenous bicortical iliac crest graft. Eur Spine J 21:156-164, 2012

11. Nout YS, Mihai G, Tovar CA, Schmalbrock P, Bresnahan JC, Beattie MS: Hypertonic saline attenuates cord swelling and edema in experimental spinal cord injury: A study utilizing magnetic resonance imaging. Crit Care Med 37: 2160-2166, 2009 
12. Okawa A, Sakai K, Hirai T, Kato T, Tomizawa S, Enomoto M, Kawabata S, Takahashi M, Shinomiya K: Risk factors for early reconstruction failure of multilevel cervical corpectomy with dynamic plate fixation. Spine (Phila Pa 1976) 36: E582-587, 2011

13. Rehman L, Mohammad Hashim AS, Qayoom Khan HA, Arain $\mathrm{SH}$, Ali S, Sheikh HA: Anterior decompression and fixation with Webb-Morley procedure in dorsolumbar spinal injury. J Coll Physicians Surg Pak 23: 330-333, 2013

14. Sayana MK, Jamil H, Poynton A: Cervical laminoplasty for multilevel cervical myelopathy. Adv Orthop 2011:241729, 2011
15. Son S, Lee SG, Park CW, Kim WK: Combined open door laminoplasty with unilateral screw fixation for unstable multi-level cervical stenosis : A preliminary report. J Korean Neurosurg Soc 53: 83-88, 2013

16. Uehara M, Takahashi J, Ogihara N, Hirabayashi H, Hashidate $\mathrm{H}$, Mukaiyama K, Shimizu M, Kato H: Cervical pedicle screw fixation combined with laminoplasty for cervical spondylotic myelopathy with instability. Asian Spine J 6: 241-248, 2012

17. Yukawa $Y$, Kato $F$, Ito $K$, Horie $Y$, Hida T, Nakashima $H$, Machino M: Placement and complications of cervical pedicle screws in 144 cervical trauma patients using pedicle axis view techniques by fluoroscope. Eur Spine J 18: 1293-1299, 2009 